\title{
Boundary Dislocations in Coincidence Site Lattice Grain Boundary*
}

\author{
By Yoichi Ishida**
}

\begin{abstract}
Fine regular structures are occasionally visible in the grain boundary of an annealed Fe-Mn alloy when observed in a highvoltage electron microscope. They are in the form of either an array of dislocations or serrations in the grain boundary. The orientation relationship between the neighboring grains was near one of the coincidence relationships and the grain boundary plane corresponded to one of the densely packed planes of the coincidence site. The structure and possible behavior of such dislocation array in the coincidence boundary were considered theoretically.

It was concluded that the Burgers vector of the boundary dislocation makes up a three-dimensional lattice which depends on the coincidence system but is independent of the boundary plane orientation. Each boundary dislocation is generally bound to a step in the grain boundary whose height depends on the boundary plane on which the dislocation is located. The Burgers vectors of lattice dislocation form superlattice with the Burges vectors of the grain boundary dislocation. Therefore, a lattice dislocation on arriving at the grain boundary disintegrates into grain boundary dislocations if the temperature is high and grain boundary diffusion is active. The Burgers vector of some of the grain boundary dislocations are parallel to the coincidence grain boundary. However, the Peierls stress for the gliding of the boundary dislocation appears to be generally high.
\end{abstract}

(Received October 3, 1969)

\section{Introduction}

A coincidence site lattice grain boundary (coincidence boundary) is an ordered boundary, many lattice, sites on which are common to both grains so that good atomic fitting is expected between the grains. A twin boundary, for example, is one of the coincidence boundaries except that it gives the best fit amongst the coincidence systems. Ranganathan ${ }^{(1)}$ and Friedel ${ }^{(2)}$ have analyzed the geometry of the coincidence systems in cubic crystals. Grain boundaries with a high density of coincidence lattice sites are known to behave differently from usual boundaries in certain properties such as secondary recrỳstallization ${ }^{(3)}$ and grain boundary migration(4). Brandon ${ }^{(5)}$ proposed that boundary dislocations allow a high degree of coincidence to persist across a grain boundary whose axes of misorientation and angular misorientation deviate from the exact coincidence. In cubic crystals, a high proportion of the boundaries was considered to contain such regular structures ${ }^{\dagger}$. Bollmann ${ }^{(6)}$ extended the concept of the coincidence model and developed a continuous form of mathematical description, " 0 lattice". In the framework of this theory the Burgers vector of grain boundary dislocation was defined by DSC lattice. A more direct definition on the Burgers vector is given in the present work.

* This paper was presented at the Autumn Meeting of the Japan Institute of Metals, Oct., 1968, Sendai, Japan. Published originally in Japanese in J. Japan Inst. Metals, 33 (1969), 1129.

** Institute of Industrial Science, University of Tokyo, Tokyo, Japan.

(1) S. Ranganathan : Acta Cryst., 21 (1966), 197.

(2) J. Friedel : Lecons de Crystallographie, Paris. referred in (1).

(3) M. L. Kronberg and F. H. Wilson : Metals Trans, 185 (1949), 501.

(4) K. J. Aust and J.W.Rutter : Trans. Met. Soc. AIME, 215 (1959), 119, 820

(5) D. G. Brandon : Acta Met., 14(1966), 1479.

$\dagger$ A separate experimental report is being prepared by the author on the proportion of the boundaries to be described by this model. The actual proportion should be higher than that calculated on the basis of random nucleation.

(6) W. Bollmann : Phil. Mag., 16(1967), 363, 383.
Some of the boundary dislocations are identical to changes in the ledge spacing of atomic planes if the actual arrangement of atoms near the core of the dislocation is assumed according to the model of Bishop and Chalmers ${ }^{(7)}$. Such an assumption in regard to the atomic arrangement is unnecessary in the present analysis.

A direct observation of the boundary dislocation was not easy with a field-ion microscope ${ }^{(8)}$, but was easy with a transmission electron microscope provided the spacing between the dislocations is sufficiently wide. Some years ago, the author analyzed statistically the Burgers vector of dislocation images on the grain boundary of a lightly strained Fe-Mn alloy foil in a $100 \mathrm{kV}$ electron microscope, and found that most of them are lattice dislocations lying on the grain boundary ${ }^{(9)}$. They disappeared on annealing the foil at elevated temperatures ${ }^{(10)}$. Recent analysis with the annealed specimen, however, revealed that regular arrays of the images are occasionally seen in the grain boundary(11). The dark field micrographs indicated that the images were probably caused by dislocations. The orientation relationships of such a grain boundary were analyzed and found to fulfil those of an important coincidence system.

This paper presents a few examples of the image observations by a high voltage electron microscope and illustrates what kind of Burgers vector can exist in the coincidence boundary and what kind of behavior is expected with the dislocation under the stress. Relations between the grain boundary step and the boundary dislocation, reactions between the lattice dislocation and the

(7) G. H. Bishop and B. Chalmers : Scripta Met., 2 (1968), 133

(8) D. G. Brandon, B. Ralph, S. Ranganathan and M.S. Wald : Acta Met., 12 (1964), 813.

(9) Y. Ishida and M. Henderson-Brown : Acta Met., 15 (1967), 857.

(10) Y. Ishida, T. Hasegawa and F. Nagata : Proceedings of the International Conference on the Strength of Metals and Alloys, Supplement to Trans. JIM, Vol. 9, (1968), p. 504.

(11) Y.Ishida, T. Hasegawa and F. Nagata : J.Appl. Phys, 40 (1969), 2182. 
boundary dislocation, the Peierls force against gliding of the boundary dislocations, etc. are also analyzed.

\section{Material and Experimental Procedure}

The material used in this experiment was a $\mathrm{Fe}-0.75 \%$ $\mathrm{Mn}$ alloy whose detailed composition is listed elsewhere ${ }^{(12)}$. Rolled sheet specimens $0.3 \mathrm{~mm}$ thick were annealed in vacuum at $850^{\circ} \mathrm{C}$ for $30 \mathrm{~min}$ and were cooled in the furnace. The resulting grain size was $0.014 \mathrm{~mm}$. The specimens were then thinned electrolytically and were examined in a Hitachi $500 \mathrm{kV}$ electron microscope. Orientation relationships of adjoining grains were determined from the selected area diffraction of the two grains. The accuracy of the orientation of the grains so determined was 4 degrees in the direction parallel to the electron beam and one degree in the angle of rotation about the axis parallel to the electron beam even when only a thick part of the foil was analyzed. Rotations to produce the orientation relationship of the two grains were calculated by an electric computer, and the data were compared with those of important coincidence systems.

\section{Transmission Micrograph Image of Boundary Dislocation}

Photo. 1 (a) is an example of the fine structure in the grain boundary. A grain boundary appears as a strip since it intersects the foil at its upper and lower surfaces. Images parallel to the strip are thickness fringes. An array of slightly curving lines diagonal to the strip is the image in question. They were due to lattice distortions and were not simply images of boundary steps or precipitates since the image appeared in the dark field micrograph and did disappear for a certain diffraction. The images were not Moirê lines since the same image could reappear when the specimen was tilted and another diffraction condition was achieved. Photo. 2(a) is another example of the dislocation array. A cyclic arrangement is visible in the array. The probability of occurrence of fine images on such a grain boundary was a few percent with this alloy. Areas were selected where the thickness fringes were fine and discrete. Sometimes the images

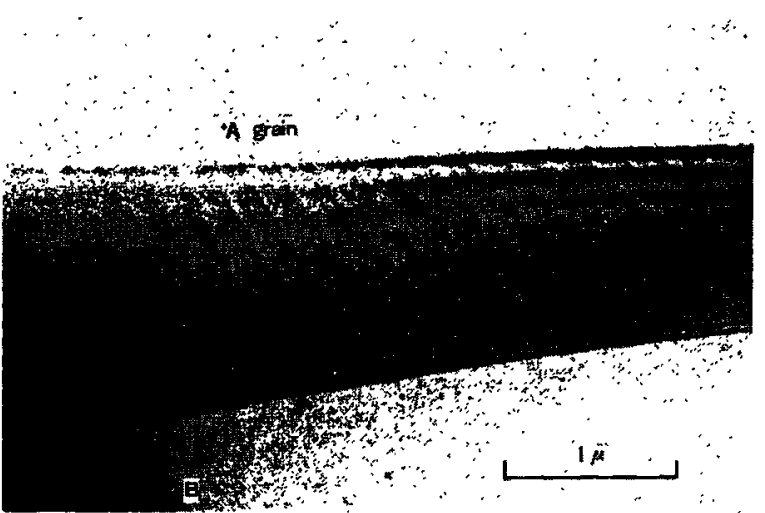

Phate. 1 (a) Atranmission electron micrograph of a grain boundary of an annealed $\mathrm{Fe}-0.75 \% \mathrm{Mn}$ alloy. An array of grain boundary dislocations is observed in addition to diffraction fringes

(12) Y. Ishida and D. Mclean : J. Iron Steel Inst., 205 (1967), 88. were visible in the micrograph but not on the fluorescent screen. About 20 grain boundaries have been observed to contain the fine structures.

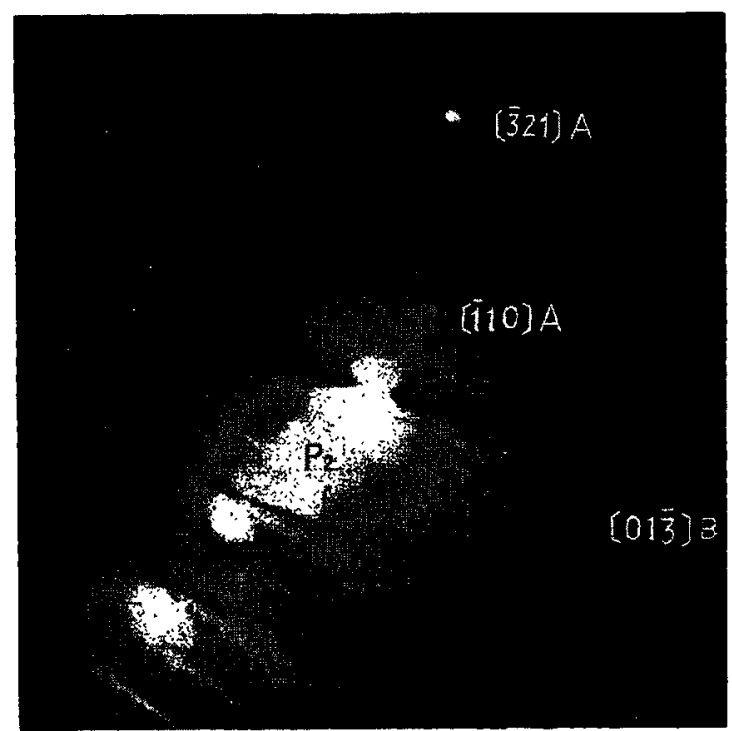

Photo. 1 (b) Composite diffraction pattern with the microscope aperture aligned on the grain boundary of Photo. 1(a)

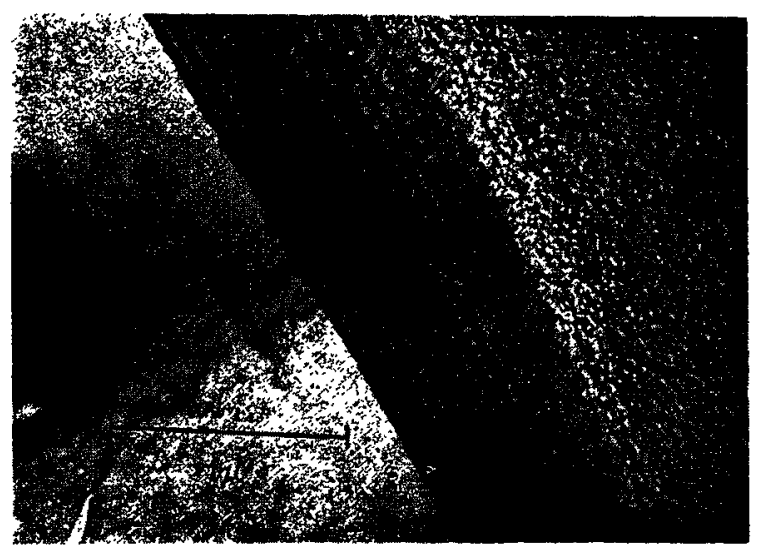

Photo. 2 (a) A cyclic array of grain boundary dislocations

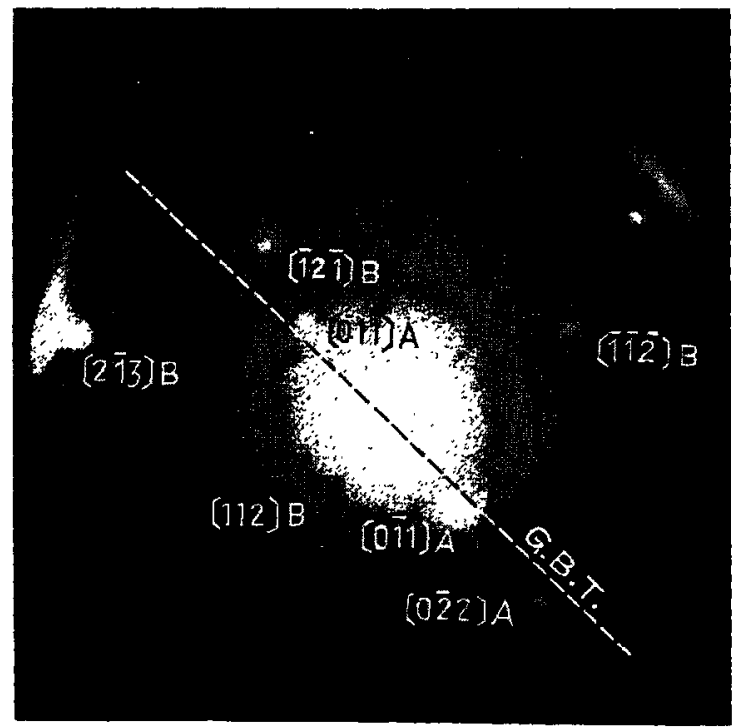

Photo. 2 (b) Selected area diffraction pattern with the aperture aligned on the grain boundary of Photo. 2 (a) 


\section{Determination of the Kind of Coin- cidence and Estimation of the Boundary Plane Orientation}

A composite pattern of the selected area diffractions (Photo. 1 (b)) was examined and approximate orientations of the neighboring grains $A$ and $B$ were determind. Spots $P_{1}$ and $P_{2}$ were the results of successive diffractions in grains $A$ and $B$. They did not appear on diffraction patterns taken separately on A and B grains. Photo. 1(b) does not show a complete (111) pattern for grain A, but diffraction patterns taken in the immediate neighboring area in grain $A$ gave perfect (111) patterns. Therefore, the [111] A was nearly parallel to the electron beam. The following approximate orientation relation was found with respect to the electron beam direction:

electron beam $/ /[111] A / /\left[\begin{array}{lll}11 & 31\end{array}\right] B$

electron beam $\perp[\overline{29} 254] \mathrm{A} / /[\overline{1} 32] \mathrm{B}$

Fig. 1 is a stereographic triangle in which closed circles

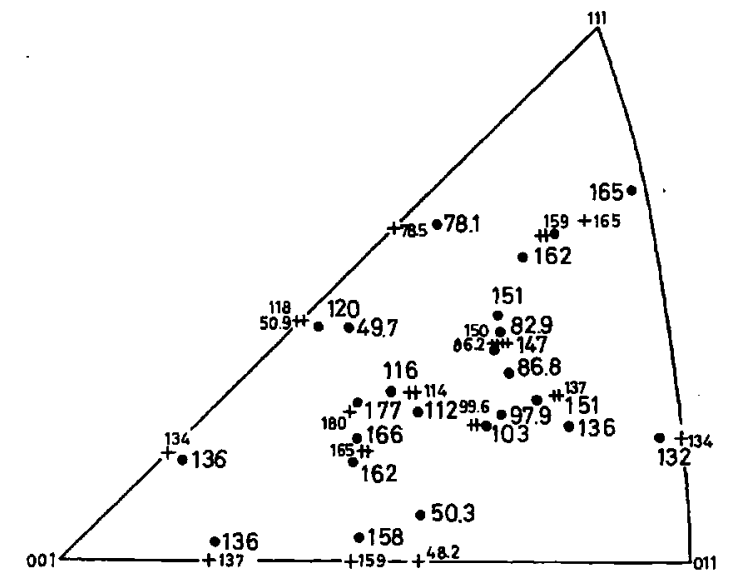

Fig. I Twenty-four axes and angles to produce the orientation of grain B from that of grain A in Photo. 1. Attached numbers are rotation angles. Crosses are those of the $\Sigma 15$ coincidence relationship. They agree to each other within the accuracy of the experiment

indicate the orientations of the rotation axes to produce the orientation of one grain from that of the other grain. The number attached to the closed circle is the angle of rotation about the axis. Since the $\mathrm{Fe}-\mathrm{Mn}$ alloy is cubic in structure any one of the 24 rotations can account for the orientation relationship. Cross marks, on the other hand, indicate those of $\Sigma 15$ coincidence system $^{(8)}$. The closed circle and cross marks agree with each other within the accuracy of the orientation determined by the diffraction pattern, suggesting the grain boundary of Fig. 1 to be a $\Sigma 15$ coincidence boundary. A similar analysis on the grain boundary of Photo. 2 (a) indicated that the grain boundary was a $\Sigma 9$ coincidence boundary. Photo. 2 (b) is the composite pattern with the microscope aperture aligned on the grain boundary of Photo. 2 (a). Spot [011] A was not observed in the photograph, but appeared in diffraction patterns taken in the neighboring area in grain $A$. Therefore, the [100] A was nearly parallel to the electron beam. A broken line indicates the orientation of the intersection line between the grain boundary and the foil surface (G. B. T.), which was measured from the multiple dark field images.
The angle $\theta$ between the normal of the grain boundary plane and that of the foil surface is measured from the kind and the apparent spacing of the thickness fringes. In Photo. 2 (a), the apparent spacing of the $\{110\} \mathrm{A}$ diffraction fringes was $560 \AA$, while each fringe corresponds to $4.25 \AA^{*}$ difference in depth from the foil surface along the beam direction. Therefore :

$$
\theta \approx \tan ^{-1} 425 / 560 \approx 37.2^{\circ}
$$

Fig. 2 is a stereograph of a $\Sigma 9$ coincidence system with

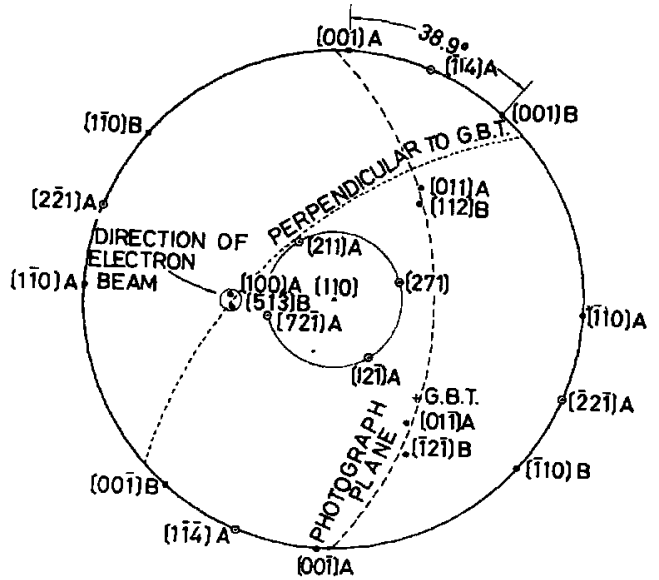

Fig. $2[110]$ stereograph of the $\Sigma 9$ coincidence grain boundary. [100] $A$ and [51 3 ] B were parallel to the electron beam in Photo. 2. The boundary plane was estimated to be a densely packed plane of the coincidence sites (211) A

$[110]$ in the center of the figure. Double circles are normals of densely packed planes of the coincidence sites. The $\Sigma 9$ coincidence system is convenient for the analysis since the relationship may be expressed by a rotation about a simple axis [110]. Closed circles along the broken line [112] $B,[01 \overline{1}] A$ and $[\overline{1} 2 \overline{1}] B$ are orientations of representative spots in the composite diffraction pattern of Photo. $2(\mathrm{~b})$. They are perpendicular to the direction of the electron beam (approximately [100] A, [513̄] B). Grain boundary plane normal should be somewhere on the dotted line and about $37.2^{\circ}$ off the direction of the electron beam. These two conditions are fulfilled if the normal of the grain boundary was [211] A in Fig. 2, which is one of the fourth densely packed plane of the coincidence sites with the $\Sigma 9$ coincidence system. Similar analysis have been applied to the grain boundaries containing the fine structure. For each of the grain boundaries a possible coincidence system was determined.

\section{Burgers Vector of the Boundary Dislocation}

The present analysis could not determine the Burgers vector of individual boundary dislocations in Photos. 1 and 2. Burgers vector of the boundary dislocation is different for each coincidence system and have in general indices of large intergers. Therefore, a usual technique

* The depth value with $500 \mathrm{kV}$ electron acceleration was estimated from that of pure iron with $100 \mathrm{kV}$ electron acceleration $(270$. $)$. Only relativistic corrections were made. The effect of alloy element was ignored since the Mn content in the specimen is only $0.75 \%$. 
to determine the Burgers vector by searching for a diffraction condition devoid of the dislocation image, is hardly applicable unless the coincidence system of the boundary is known in advance. The Burgers vector of dislocations in a regular array may be measured indirectly by measuring the change in the boundary orientations with the array spacing. However, one finds it very difficult to apply this method unless the grain boundary is made very close to one of the coincidence relationships, because many arrays of boundary dislocations which are usually contained in the grain boundary may disappear if the array spacing is smaller than $100 \AA^{(11)}$. These difficulties may be overcome if analyses are made with the grain boundary of a bicrystal specially prepared for one of the coincidence relationships*. In the present analysis theoretical considerations are given to the possible Burgers vector of the boundary dislocation, and the expected general behavior is compared with the experimental results.

\section{Discussion}

\section{Construction of boundary dislocation}

As an example, the $\Sigma 17 \mathrm{~b}$ coincidence boundary is analyzed. The $\Sigma 17 \mathrm{~b}$ coincidence boundary has been found experimentally and analyzed in a previous paper ${ }^{(11)}$. In Fig. 3 the lattice site arrangement is described with a rotation axis [110] perpendicular to the surface of the paper. Open and closed circles indicate lattice sites of the neighboring grains. They are superimposed for the

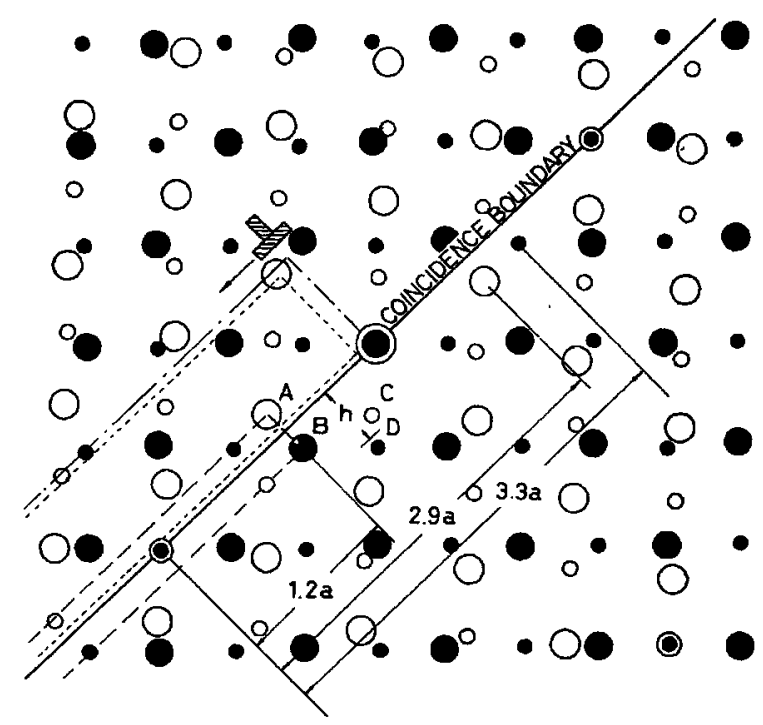

Fig. 3 Lattice sites configuration in $\Sigma 17 \mathrm{~b}$ with (110) parallel to the paper plane. White and black circles indicate lattice points of the neighboring grains superimposed to analyze the Burgers vector and the step height of the grain boundary dislocation

Lattice sites of grain

$\left.\mathrm{P}_{\mathbf{B}}^{\mathbf{A}}\right\}$ in the paper plane

A $\mathrm{A}$ in the plane one atomic layer above or

- B below the paper plane

* Bicrystals containing $\Sigma 9$ coincidence boundaries have been prepared by a repeated directional annealing method for the analysis. convenience of the analysis; once the grain boundary is decided upon in Fig. 3 , there are only the open circles. on one side of the boundary and only the closed circles on the other side. Large circles are on the surface of the paper while the small ones would lie in the plane one atomic layer above or below the surface of the paper. The two sets of atomic planes are sufficient to describe the entire crystal lattice, since the $\mathrm{Fe}-\mathrm{Mn}$ alloy is of the $b c c$ structure and stacks according to an $\mathrm{ABAB}$ sequence along the [110]. Double circles indicate the coincidence sites. The solid line is the trace of the most densely packed plane of the coincidence sites $(3 \overline{3} 4)$, which is. perpendicular to the paper. If the grain boundary is the coincidence plane, all lattice sites on the grain boundary are common to those of the neighboring grains so that the boundary energy is likely to be lower than that of a random boundary.

The grain boundary energy cannot be easily calculated since the real atomic arrangement along the grain boundary should be understood first. Minute amounts of impurity can segregate in the grain boundary and readily affect the boundary energy. The coincidence orientation relationship, however, is a necessary condition if the grain boundary energy is lower than that of the random grain boundary. The degree of saving of the boundary energy is not directly related to the density of the coincidence site, but the probability of achieving a large reduction in the grain boundary energy increases as the density of the coincidence site increases. The way to create a boundary dislocation is just like that of the lattice dislocation. Consider matching of lattices A and B in Fig. 3. Let us take out all.atoms enveloped in the broken line and rejoin the remaining two sections. An edge dislocation with the Burgers vector $b=\overrightarrow{\mathrm{AB}}$ would be created by this process. We see that the newly produced grain houndary holds the same coincidence relationship; the edge dislocation stays in a single coincidence boundary. A similar operation on lattice sites $\mathrm{C}$ and $\mathrm{D}$ creates another boundary dislocation. In this case, however, a step of height $h$ must be introduced in the grain boundary. Otherwise, the coincidence boundary terminates at the dislocation and a high energy grain boundary extends beyond it. Therefore, energetically, there would be strong tendency to form the step at the dislocation although an extra energy is needed to form the step. The boundary step is inseparable from the boundary dislocation and is different from that proposed by Brandon ${ }^{(5) \dagger}$. The existence of the step is important since it contributes to the deviation in the orientation of the grain boundary plane from that of the exact coincidence plane.

The above argument is also valid if the dislocation contains a screw component. Fig. 4 shows some of the Burgers vectors allowed in the coincidence boundary. The figure is constructed by collecting all the matching

$\dagger$ The boundary step proposed by Brandon ${ }^{(5)}$ was simply a switch of the boundary plane from a densely packed plane of the coincidence sites to the neighboring one in a perfect "dislocation-less" coincidence boundary. The height of the step, therefore, is the spacing between the coincidence planes. A Brandon's step (without a boundary dislocation) does not have a long range strain field around it. 
of lattice point pairs on a (110) plane. A similar operation may be performed between the lattice point of different (110) planes. Therefore, the Burgers vector lattice is in fact three-dimensional. Each coincidence system defines its own lattice of the Burgers vector, which is independent of the orientation of the coincidence boundary. The height of the boundary step $h$, however, depends on the coincidence boundary selected. The Burgers vector lattice is essentially the same as the DSC lattice of Bollmann ${ }^{(6)}$, except that the latter was defined

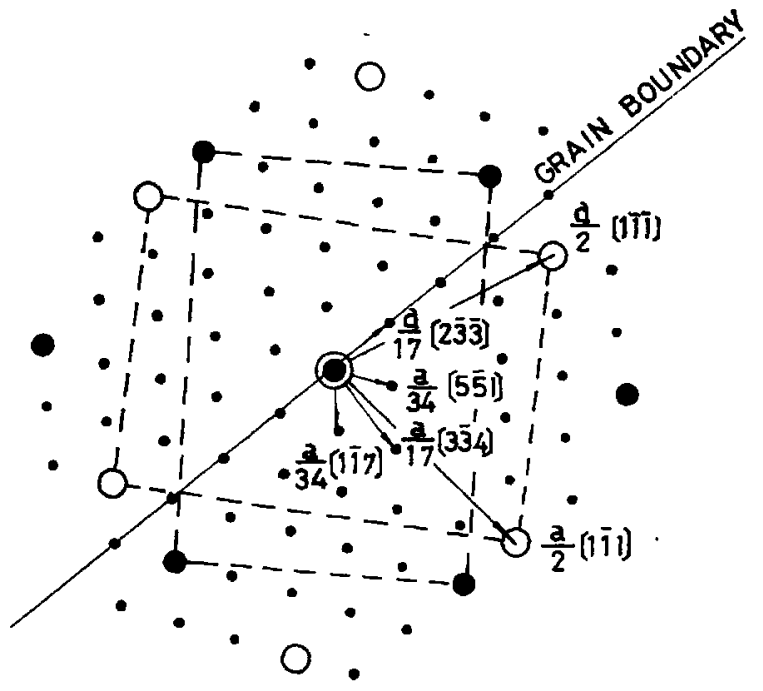

Fig. 4 Burgers vectors of $\sum 17 \mathrm{~b}$ grain boundary dislocations in (110) constructed from Fig. 3. Large open and closed circles coincide with the Burgers vector of lattice dislocations

as two-dimensional translations which preserve butterfly patterns in his 0 lattice. The definition adopted in the present analysis is more direct and general than that proposed by Bollmann.

Although the above analysis concerns with a $\Sigma 17 \mathrm{~b}$ coincidence boundary, the same procedure is applicable to all the other coincidence boundaries.

\section{Grain boundary dislocation array and transition between the neighboring coincidence systems}

Another $\Sigma 17$ b coincidence boundary may be produced when in the boundary of Fig. 3 an array of boundary dislocations with $b=a / 17[334]^{*}$ are superimposed at intervals of $2.9 \mathrm{a}$. In case the dislocations of the reverse sign are superimposed with the array spacing of $1.2 a$, the $\Sigma 3$ coincidence boundary (twin boundary) is formed; any coincidence boundary can transform into that of the neighboring coincidence system if a sufficient amount of the boundary dislocation is supplied. A major problem arises as to whether or not the grain boundary possesses a regular structure for the intermediate orientations between the two coincidence systems. Bishop and Chalmers ${ }^{(7)}$ assumed such regularity all over the range. Another interesting problem is the existance of coincidences of higher $\Sigma$. For example, a $\Sigma 43$ boundary instead of $\Sigma 3$

* This dislocation may not be stable and decompose if boundary steps are allowed: $a / 17[3 \overline{3} 4] \rightarrow a / 34[5 \overline{5} 1]+a / 34[1 \overline{1} 7]$. The general argument, however, is not affected. can be formed if the dislocation array spacing is $3.3 a$ instead of $1.2 a$. Unfortunately, electron microscopy cannot reveal a dislocation array when the spacing is less than a hundred $\AA$.

\section{Decomposition of lattice dislocations into grain boundary dislocations}

In Fig. 4, the large open and closed circles indicate the Burgers vectors of lattice dislocations of the neighboring grains. They correspond to a super-lattice of the Burgers vector of the boundary dislocation. Therefore, any lattice dislocation which has arrived at the grain boundary can decompose into several boundary dislocations if the temperatures is high and the grain boundary diffusion is active.

For example;

$$
\begin{aligned}
a / 2[1] \overline{1}] \rightarrow & a / 34[5 \overline{5} 1]+a / 17[2 \overline{2} \overline{3}] \\
& +a / 17[2 \overline{2} \overline{3}]+a / 17[2 \overline{2} \overline{3}] \\
a / 2[1 \overline{1} 1] \rightarrow a / 34[5 \overline{5} 1]+a / 34[5 \overline{5} 1] & \rightarrow a / 34[5 \overline{5} 1]+a / 34[1 \overline{1} 7]+a / 34[1 \overline{1} 7]
\end{aligned}
$$

Similar decomposition reactions are expected with the other coincidence systems.

The boundary dislocations considered here are only perfect dislocations. Imperfect dislocations can also exist, since the stacking fault is likely to occur in the grain boundary. Dislocation images disappeared from the grain boundary of a lightly strained Fe-Mn alloy on annealing the foil in an electron microscope ${ }^{(10)}$. Since those dislocations were mainly lattice dislocations ${ }^{(9)}$, they are likely to have decomposed into the boundary dislocations, which can then migrate ${ }^{\dagger}$ and annihilate if boundary dislocations of opposite sign pre-existed, or just lose their long range strain field if they join the dislocation array pre-existed along the grain boundary. The microscopic image disappears when their long range strain fields vanish.

\section{Grain boundary sliding due to the boundary dislocation}

As shown in Fig. 3 there is always a boundary dislocation whose Burgers vector is parallel to the coincidence boundary plane. Crussard and Friedel ${ }^{(13)}$ proposed that such boundary dislocations occur by the decomposition of a lattice dislocation on the grain boundary, glide along the grain boundary, multiply like a Frank-Reed source and cause grain boundary sliding in creep. Recently, Gleiter et al. ${ }^{(14)}$ observed dislocation gliding along a grain boundary in a $\mathrm{Ni}-\mathrm{Al}$ alloy foil and suggested that they are boundary dislocations whose glide plane is the grain boundary and the Burgers vector lies within the grain boundary; no step is connected with the dislocation. On a coincidence tilt boundary such a dislocation should have a large Burgers vector as $b=a[\overline{2} 2 \overline{3}]$ in Fig. 3 . The dislocation would be unstable and decompose into 17

$\dagger$ Such a long range movement of the grain boundary dislocation have been observed by transmission electron microscopy at elevated temperatures.

(13) C. Crussard and J. Friedel : Creep and Fracture of Metals at Elevated Temperatures, H.M. S. O., London, (1956), p. 243.

(14) H. Gleiter, E. Hornbogen and G. Bäro : Acta Met., 16 (1968), 1053. 
boundary dislocations, $b=a / 17[\overline{2} 2 \overline{3}]$ as described in Fig.4, if the temperature is high and the grain boundary diffusion is active. When the $a / 17[\overline{2} 2 \overline{3}]$ dislocation glides parallel to the grain boundary downwards to the right in Fig. 3, atoms within the dotted line have to shuffle*. Since the shuffling process is limited within the core of the dislocation and does not involve a long range atomic diffusion, the activation energy of the process is not as high as that of self-diffusion, but it would contribute as the high Peierls force against the gliding of the boundary dislocation if the step of the boundary dislocation is high and the shuffling becomes extensive. Gliding of the boundary dislocation may be easier for twist boundaries because many boundary dislocations exist without steps. Assume, for example, that the grain boundary plane was the paper plane of Fig. 3. All the boundary dislocations with the Burgers vector in Fig. 4 are step-free, so that the required atomic shuffle on dislocation glide is confined within the boundary. The Peierls force, however, still appears to be high since the boundary plane is usually not the slip plane of the crystal.

The situation is very different with the twin boundary ( $\Sigma 3$ coincidence boundary), because the boundary plane is also the slip plane of the crystal. If the grain boundary observed by Gleiter et al. was a twin boundary of Al-Ni alloy $(F C C)$, a Shockley partial dislocation $b=a / 6$ $[11 \overline{2}]$ in Fig. 5 corresponds to the boundary dislocation

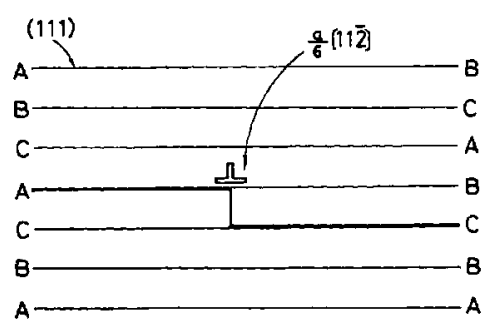

Fig. 5 A Shockley partial dislocation in the twin boundary of a FCC structure. A, B and C on both ends of the horizontal lines indicate the way of (111) stacking. The boundary shifts by one atomic plane as the dislocation glides along the boundary

with a step. The rearrangement of atoms on passing the Shockley dislocation is very minor and the dislocation glides easily as is generally known.

There is evidence that the observation of Gleiter et al. ${ }^{(14)}$ was on the gliding of Shockley partial dislocations although the orientation relationship is not analyzed in their paper; in their Fig. 4, two grain boundaries are straight and run parallel to each other. The thickness fringes in one boundary are a mirror image of those in the other. The appearance of the matrix is very similar again across the two boundaries. Moreover, in Fig. 4 (c), a slip trace in the grain is parallel to the two boundaries. These features may be explained by the assumption that the two boundaries are a pair of annealing twin boundaries and

* Near the grain boundary the actual position of the atoms do not coincide with the lattice sites. The shuffling of atoms, nevertheless, would occur with the movement of the boundary step. The grain boundary migrates as the step moves. Grain boundary migration is generally found to occur with the sliding during creep of metals at elevated temperatures.
Shockley partial dislocations $(b=a / 6[11 \overline{2}])$ glide instead of step-free boundary dislocations (for example $b=a / 2$ [10I]). In the present experiment, the thermal stress due to the electron beam did not cause any detectable gliding to the boundary dislocation. Movement of the dislocation image was not noticed even when the foil was made from a lightly strained specimen and heated in the electron microscope ${ }^{(10)}$. Further experiments are needed to investigate whether or not the discrepancy in the glide behavior of the boundary dislocation is due to the difference in the alloy used for the experiments.

\section{Conclusion}

(1) Grain boundaries of an annealed $\mathrm{Fe}-0.75 \% \mathrm{Mn}$ alloy were observed by transmission electron microscopy. Boundaries with an array of dislocation images were analyzed. The orientation relationship between the neighboring grains agreed with one of the coincidence relationships within the accuracy of the experiment. The grain boundary plane corresponded to one of the densely packed plane of the coincidence sites.

(2) The Burgers vector of the boundary dislocation makes up a three-dimensional lattice which depends on the coincidence system but is independent of the boundary plane orientation. Each boundary dislocation is generally bound to a step in the grain boundary, whose height depends on the boundary plane on which the dislocation is located.

(3) The array of boundary dislocations allow the orientation of the neighboring grains to deviate from that of the exact coincidence. The orientation of the grain boundary plane may deviate from a densely packed plane of the coincidence site by containing an array of the boundary dislocations with step or by combination with other densely packed planes of the coincidence.

(4) The Burgers vector of the lattice dislocation forms a superlattice with the Burgers vector of the grain boundary dislocation. The lattice dislocation on arriving at the grain boundary will disintegrate into grain boundary dislocations if the temperature is high and the grain boundary diffusion is active.

(5) Some of the Burgers vectors of the grain boundary dislocations are parallel to the coincidence grain boundary. However, the Peierls stress for the gliding of the boundary dislocation generally appears to be high. In this experiment, the thermal stress due to the electron beam did not cause any detectable gliding of the boundary dislocations.

\section{Acknowledgment}

The author thank Mr. T. Hasegawa at Showa Denko Central Research Laboratory for his cooperation with the electron microscopy experiments, Mr. K. Sasa, graduate student at University of Tokyo for his help in computer programing, Prof. T. Suzuki at Institute for Solid State Physics, University of Tokyo for permitting the use of a Hitachi $500 \mathrm{kV}$ electron microscope and Sakkokai Foundation for a fellowship grant to the author. 